Revista de Psicología de la PUCP. Vol. XXII, 1, 2004

\title{
Evaluación de la estructura factorial del Cuestionario de Valores Interpersonales (SIV)
}

\author{
César Merino Soto ${ }^{1}$ \\ Defensoría Municipal del Niño y del Adolescente
}

\author{
Dean W. Wichern ${ }^{2}$, Karin Rivas ${ }^{3}$ \\ Texas A\&M University
}

El artículo evalúa la estructura factorial bajo los efectos del método ipsativo de respuesta, estudiados en el nivel de las subescalas del Cuestionario de Valores Interpersonales de Gordon (SIV), y las relaciones entre ellas, en una muestra de adolescentes entre 15 y 17 años de ambos sexos y procedentes de un colegio privado y estatal, representativos de los niveles socioeconómicos medio y bajo. Aunque el SIV ha sido una herramienta extensamente utilizada, no se reportado previamente un análisis de su estructura factorial en muestras Latinoamericanas. Mediante el análisis de componentes principales y el análisis factorial con un enfoque confirmatorio, se han identificado relaciones bipolares entre Independencia y Benevolencia, y Soporte y Conformidad. Se obtuvo también la confirmación del modelo de valores interpersonales propuesto por L.V. Gordon. En el análisis se consideró un aspecto que artificialmente puede haber influido en el patrón de correlaciones entre los componentes, esto es el método ipsativo de las preguntas del SIV. Finalmente, se discute sobre las medidas ipsativas y sus consecuencias en la interpretación de sus resultados.

Palabras clave: Cuestionario de Valores Interpersonales de Gordon, análisis factorial, análisis de componentes principales, medidas ipsativas.

\section{Assessment of the factorial structure of the Survey of Interpersonal Values}

The present study evaluates the factorial structure, in the level of the subscales, of the Survey of Interpersonal Values (SIV), and the relationships among them, in a sample of adolescents between 15 and 17 years old of both sexes and from a private and public school, representative of low and middle socioeconomic levels. Although the SIV has been a widely used tool, there is no report of an analysis of its factorial structure in Latin-American samples. By means of the principal components analysis and the factorial analysis with a confirmatory approach, bipolar relationships have been identified between Independence and Benevolence, and Support and Conformity. The confirmation of the pattern of interpersonal values proposed by $\mathrm{L}$. V. Gordon was also accomplished. An aspect considered in the analysis may have artificially influenced the pattern of correlations among the components, and the reliability: the ipsative method of the questions of the SIV. Finally, there is a discussion about the ipsative measurement and its consequences in the interpretation of the results.

Key words: Survey of Interpersonal Values of Gordon, factorial analysis, principal component analysis, ipsative measure. 
Desde los años setenta y específicamente en el contexto peruano, el SIV ha sido favorecido por el interés de varias investigaciones centradas en sujetos adolescentes y adultos, tanto normales como con algún tipo de psicopatología, por ejemplo, consumidores de droga (Vela, 1989). En los estudios peruanos, las muestras sobre las que se han recolectado los datos han sido variadas; por ejemplo, adolescentes (Núñez, 1973; Castillo, 1977; Rivas, Wichern \& Merino, 2002), adultos obreros (Silva, 1982), adultos con psicopatología (Vela, 1989) y universitarios (Crocé, 1985). En estas investigaciones, el enfoque aplicado se orientó al uso de las subescalas como variables dependientes o variables de respuesta (Hutcheson \& Sofroniou, 1999).

Recientemente, el SIV se ha utilizado para evidenciar los aspectos del comportamiento relacionados a la conducta asertiva. En un análisis factorial exploratorio, Bouvard et al. (1999) refieren que las escalas del SIV se agruparon en componentes bipolares y ortogonales al estudiar sujetos de control normales, a los que se les aplicó también medidas de síntomas psicopatológicos como el Inventario de Depresión de Beck (Beck et al., 1979), el Mini-Mult (Kincannon, 1968) y el cues-

I Psicólogo graduado de la Universidad Inca Garcilaso de la Vega. Jefe del Servicio de Psicología de la Defensoría Municipal del Niño y del Adolescente. Sus intereses se orientan a la prevención del maltrato infantil y de los problemas de ajuste social en los colegios, la metodología psicométrica y la aplicación de métodos multivariados en el análisis de datos psicológicos. Correo electrónico: sikayax@yahoo.com.ar

2 Profesor John E. Pearson de Administración de Negocios en Texas A\&M University. Autor y coautor de numerosos artículos científicos, y coautor de cuatro libros de estadística multivariada. Sus intereses de investigación son la aplicación de métodos estadísticos en varias disciplinas, el análisis de series de tiempo, métodos multivariados y modelos lineales. Correo electrónico: d-wichern@tamu.edu

3 Psicóloga graduada de la Universidad San Martín de Porres, dedicada a la práctica profesional privada. Sus intereses de investigación abordan el ajuste social de los estudiantes en el contexto escolar. 
tionario de temores (Marks \& Mathews, 1979). Pero esta bipolaridad entre las escalas de SIV parece ser una característica distintiva de las medidas que tienen métodos ipsativos en su calificación. (Loo, 1999; Weiss, 1970).

Aunque a través de los años el uso continuado del SIV lo ha hecho un instrumento conocido entre los clínicos e investigadores, menos conocido ha sido el impacto que tiene su formato de respuesta en el análisis correlacional; esto es, su formato ipsativo. Las medidas ipsativas han sido adoptadas desde hace muchos años por instrumentos de personalidad como el Cuestionario de Preferencias Personales de Edwards (Helms, 1983), el Inventario de Estilos de Aprendizaje de Kolb (1976) o la Escala de Tendencias a la Acción en Niños (Deluty, 1979). Pero las medidas con características ipsativas han sido cuestionadas desde que aparecieron y generalmente están acompañadas de precauciones sobre su interpretación y métodos de análisis ejecutados sobre los datos (Gregory, 2000; Guilford, 1952; Loo, 1999; Weiss, 1970, 1971). Pero aún con todo, parece que la "elegancia" de este formato (Gregory, 2000) y otros aspectos considerados por el usuario lo hacen elegible para su uso continuado actualmente. El debate aún está vivamente abierto y no está completamente resuelto (Cornwall \& Dunlap, 1994; Saville \& Willson, 1991). Como señalan Van Gelderen y Frese (1998), la medición ipsativa tiene ventajas y desventajas. Entre las ventajas están que los sujetos son forzados a hacer deliberadas comparaciones simultáneas entre las dimensiones evaluadas. Adicionalmente, la deseabilidad social tiende a reducirse. Entre las desventajas que señalan Van Gelderen y Frese (1998) se tiene que las respuestas no son independientes una de otra, lo que afecta adicionalmente a las correlaciones entre los ítems o subescalas. Los análisis con estos datos tienden a tener problemas orientados en la interpretación, pues los ítems o subescalas tienden a formar patrones de correlaciones que están influenciados justamente por el método ipsativo de respuesta. 
Evaluación de la estructura factorial del Cuestionario de Valores Interpersonales (SIV)

Cuando se examinan los patrones de correlaciones entre las variables (por ejemplo, ítems o subescalas), generalmente son abordados por el enfoque analítico factorial, que es uno de los métodos más reconocidos para abordar los patrones de correlaciones (Heise \& Bohrnstedt, 1970). Heise y Bohrnstedt (1970) señalan que la evaluación de la validez de una medida debe ser abordada por un enfoque factorial que describa las relaciones subyacentes entre las mediciones observadas (puntajes directos obtenidos) y los atributos psicológicos latentes. Estos últimos no son medidos directamente y los instrumentos de medición son, por lo tanto, un medio para acercarnos a identificar la estructura subyacente a los puntajes y explicarnos sus relaciones.

Considerando el SIV, hay algunas preguntas que se deben resolver: ¿sus puntajes están relacionados o esencialmente miden seis conductas independientes? En términos estadísticos podríamos preguntar: ¿los puntajes están altamente correlacionados o las correlaciones entre ellas son insignificantes y de baja magnitud? De manera equivalente, podemos preguntar: ¿las variables originales representan los mismos constructos subyacentes? Finalmente, ¿el formato ipsativo de respuesta influencia las correlaciones entre los puntajes? El presente estudio persigue las respuestas concernientes a la estructura teórica del SIV (y por lo tanto a la validez de constructo) y los efectos artificiales del método ipsativo de respuesta.

Si las $p$ variables están interrelacionadas, entonces podríamos construir un número reducido de $m$ nuevas variables provenientes de las variables existentes, que no estén correlacionadas, pero que aún recojan la variabilidad (es decir, retengan la información) de las variables originales. Adicionalmente, podríamos hacer una interpretación del contenido de las nuevas variables halladas. Esto último es el objetivo del llamado análisis de componentes principales (ACP).

Otro enfoque para efectuar la reducción del número de variables y su interpretación se inicia con otra pregunta: ¿las variables 
originales representan los mismos constructos subyacentes? Es decir, ¿las variables miden lo mismo (o diferentes aspectos de lo mismo) que dicen medir? Si es así, podemos tener un modelo descriptivo de lo que subyacentemente ocurre entre los puntajes e interpretar los datos multivariados en términos de unas pocas entidades no observables, llamadas factores (que en nuestra investigación son valores interpersonales). Este aspecto nos recuerda el proceso de validación de constructo requerido para enriquecer la validez de un instrumento (Cronbach \& Meehl, 1955; Nunnally \& Bernstein, 1995). Aquí entramos al tema del análisis factorial propiamente dicho, que está orientado por el siguiente argumento: supongamos que las variables pueden ser agrupadas a partir de sus correlaciones, esto es, supongamos que todas las variables dentro de un grupo en particular están altamente correlacionadas entre ellas mismas, pero tienen correlaciones relativamente pequeñas con variables provenientes de un grupo diferente. Entonces, es concebible que cada grupo de variables represente un simple factor subyacente que es responsable de las correlaciones observadas. Por ejemplo, un grupo de puntajes de una prueba de ciencias sociales, lenguaje, matemáticas y lógica, podría sugerir que hay un factor subyacente que se podría asumir sea "inteligencia". Este es el tipo de estructura que el análisis factorial intenta modelar.

Se podría considerar al análisis factorial como una extensión del análisis de componentes principales, y se puede ver a ambos como intentos para explicar las covarianzas (o correlaciones) de las variables originales en términos de un menor número de variables no correlacionadas u ortogonales (Johnson \& Wichern, 2002). Sin embargo, el análisis factorial es más elaborado. La pregunta primaria en el análisis factorial es si los datos son consistentes con una estructura conocida, prescrita como un modelo factorial ortogonal u oblícuo. La elección de los procedimientos para extraer factores ha creado cierta confusión entre los usuarios. Lo cierto es que existen diferencias en los objetivos científicos y estadísticos, algunas formulaciones algebraicas, conceptualizaciones teóricas y soluciones finales distintas entre el análisis 
Evaluación de la estructura factorial del Cuestionario de Valores Interpersonales (SIV)

de componentes principales (ACP) y el análisis factorial (AF) (Cudeck, 2000). Generalmente, los investigadores no han hecho de esta información un fundamento para sus decisiones sobre la elección del método multivariado para reducir sus datos. Otros han encontrado que la decisión entre ACP y AF no tiene suficientes bases empíricas para preferir una sobre otra (Velicer \& Jackson, 1990). Hay un recurrente debate entre la preferencia y adecuabilidad de uso entre estos dos métodos en las ciencias conductuales, aunque parece que la tendencia metodológica se orienta hacia el análisis factorial como el método de elección para los datos psicológicos (Fabrigar et al., 1999; Velicer \& Jackson, 1990; Wegener \& Fabrigar, 2000). Nosotros valoraremos estas diferencias en el terreno aplicado de nuestra investigación, usando ambos métodos.

Los métodos de factorización deben converger cuando la estructura interna de un instrumento está bien definida por la teoría y cuidadosamente diseñada (Nunnally \& Bernstein, 1995), reflejándose en la estructura factorial como sucede con el SIV. El enfoque de su diseño fue describir los valores interpersonales vistos como constructos independientes y que la deseabilidad social de los sujetos sea reducida por medio del formato de respuesta (Gordon, 1993). Pero estudios que repliquen esta estructura en adolescentes latinoamericanos parecen ser muy poco conocidos. Un reciente intento de explorar las correlaciones entre las subescalas fue hecho por Bouvard et al. (1999), en relación a otras medidas de síntomas psicopatológicos en adultos. Pero el mérito de Bouvard y sus colaboradores parece oscurecido porque no advirtieron el efecto artificial del formato de respuesta del SIV en sus interpretaciones de las correlaciones inter-subescalas.

En nuestro estudio evaluaremos la asumida estructura subyacente de los puntajes de las escalas del SIV. Hay seis respuestas de interés: $Y_{1}=$ Independencia,$Y_{2}=$ Soporte,$Y_{3}=$ Benevolencia, $Y_{4}=$ Conformidad, $Y_{5}=$ Liderazgo y $Y_{6}=$ Reconocimiento; estos puntajes pertenecen a sus respectivas subescalas y se asume que miden independientemente 
los atributos nombrados por sus títulos; por lo tanto, nuestro objetivo concierne a la validez del SIV. Por otro lado, expondremos los efectos sobre las correlaciones entre las subescalas debido al método ipsativo de respuesta.

\section{Metodología}

\section{Participantes}

El grupo de participantes será identificado como una muestra de conveniencia, que es un grupo de casos que seleccionados debido a su disponibilidad y acceso (Gall, Borg \& Gall, 1996). Usualmente este tipo de muestra, como otras de naturaleza no probabilística, son elegidas cuando la naturaleza de la investigación es exploratoria (Gall et al., 1996); además, los resultados estadísticos y las conclusiones desprendidas desde estas muestras se pueden ver como una aproximación de los resultados obtenidos si se aplicase un diseño apropiadamente probabilístico (May, Masson \& Hunter, 1990). Así, dos colegios fueron elegidos para representar los niveles socioeconómicos (NSE) bajo y medio, sumando 130 adolescentes del último grado escolar secundario; las secciones desde las cuales provienen los adolescentes fueron elegidas al azar, todas del turno diurno. El colegio estatal del NSE bajo está ubicado en un distrito distinto al del colegio privado de los adolescentes del NSE medio. Como en otras investigaciones sociales, esta selección fue guiada por la mayoritaria tendencia de grupos socioeconómicos que asisten a estos dos colegios. Como se observa en el Cuadro 1, el NSE y el género fueron proporcionales. Respecto a la edad, casi el $60 \%$ estuvo compuesto por adolescentes de 16 años. 


\section{Cuadro 1}

Descripción de la muestra

\begin{tabular}{lccc}
\hline & & $N$ & $\%$ \\
\hline Nivel Socioeconómico & & \\
& Bajo & 72 & 55.4 \\
& Medio & 58 & 44.6 \\
\hline Edad & 15 & 20 & 15.4 \\
& 16 & 76 & 58.5 \\
& 17 & 34 & 26.2 \\
\hline Sexo & & & \\
& Varón & 62 & 47.7 \\
& Mujer & 68 & 52.3 \\
\hline Total & & 130 & \\
\hline
\end{tabular}

\section{Instrumento}

EI SIV fue construído desde el análisis factorial (Gordon, 1993). Es un cuestionario de elección forzada con 90 ítems. El SIV mide seis valores explicativos de la relación del individuo con los demás: Soporte (ser tratado con entendimiento y amabilidad), Conformidad (hacer lo que es socialmente correcto), Reconocimiento (ser admirado y reconocido), Independencia (tener el derecho de lo que uno determine), Benevolencia (ayudar a otros) y Liderazgo (tener autoridad sobre otros). Estas subescalas forman seis puntajes independientes. Para efectuar la calificación de las subescalas, todos los ítems deben ser respondidos por los sujetos, de tal modo que la suma de las respuestas a todos los ítems siempre debe ser 90. En el manual se reporta estimaciones de confiabilidad desde .78 hasta .89 (Gordon, 1993). En nuestra muestra, las estimaciones de confiabilidad han sido marginalmente aceptables y bajas. En el Cuadro 2 se observa que las confiabilidades más bajas provienen de Soporte $(\forall=.57)$ y Reconocimiento $(\forall .55)$. Las confiabilidades aceptables provienen de Independencia $(\forall=.74)$, Benevolencia $(\forall=.78)$, Conformidad $(\forall=.78)$ y Liderazgo 
$(\forall=.72)$. El comportamiento de algunos de los ítems no ha sido aceptable: el ítem 29 (Soporte) con correlación ítem-test corregida (Ritc) tuvo signo negativo y de magnitud cero; los ítems 21 y 84 (Liderazgo) tuvieron Ritc cero; también fueron cuestionables los items 19 (Ritc $=0$ ) y 77 (Ritc con signo negativo) de Reconocimiento. Sin embargo, estos ítems fueron retenidos debido a que tales correlaciones pueden haber surgido de las características idiosincrásicas de la muestra, como consecuencia del error de muestro, y no ser un efecto sistemático observable en otras muestras (Worrell, Vandiver \& Watkins, 2001).

\section{Procedimiento}

Después de conseguir la autorización de los directores de ambos colegios, la aplicación de las pruebas se efectuó en un día normal de clases. Todos los alumnos presentes el día de la evaluación respondieron a la prueba. Debido a que la puntuación de las subescalas del SIV exige que cada sujeto responda a todas las afirmaciones, se descartaron 30 protocolos de adolescentes que no cumplieron con esta exigencia. La aplicación se realizó con las instrucciones estándares para la aplicación grupal.

\section{Análisis}

Dado que los métodos analíticos factoriales requieren que la distribución de las variables estén ajustadas a la distribución normal (Johnson \& Wichern, 2002), se efectuó un análisis exploratorio de los datos. Se observó que los puntajes en Liderazgo tienden a tener un ligero desvío de la normalidad, los que nos sugiere un posible reemplazo del puntaje directo de Liderazgo por su raíz cuadrada, para acercarlo a la normalidad; pero tal procedimiento no produjo un notable cambio en el análisis de las correlaciones. 
Por otro lado, considerando la taza entre el tamaño de la muestra $(\mathrm{n}=130)$ y el número de variables (seis subescalas), la proporción es razonable dados los objetivos exploratorios del estudio (Velicer \& Jackson, 1990).

De las diferencias reconocidas por varios autores (Cudeck, 2000; Fabrigar et al., 1999; Wegener \& Fabrigar, 2000), aplicamos el análisis de componentes principales para obtener una partición de ítems en subconjuntos homogéneos, y el análisis factorial para revelar los factores subyacentes que hipotéticamente explican la ortogonalidad de las subescalas.

Empezamos con un análisis ACP basándonos en la matriz R de correlaciones de nuestra muestra de estudiantes. Se debe hacer notar que, debido a que las mediciones en todas las seis subescalas tienen la misma magnitud, un ACP de la matriz de covarianza de la muestra producirá aproximadamente los mismos resultados que un ACP de la matriz de correlaciones de la muestra. Luego del análisis de componentes principales, se aplicará el análisis factorial para buscar los factores comunes subyacentes en el SIV, y luego rotaremos las cargas (Johnson \& Wichern , 2002). Debido a que la factorización de medidas ipsativas produce problemas especiales (Loo, 1999), como una dependencia lineal exacta entre las variables estudiadas, se implementó un método que también ha sido reportado en Loo (1999): la eliminación de una de las subescalas y la rotación ortogonal varimax.

\section{Resultados}

La información básica se muestra en el Cuadro 2. Se observa que los puntajes en Independencia, Soporte y Benevolencia forman una tríada distante de las puntuaciones de Conformidad, Liderazgo y Reconocimiento. La dispersión de los datos sigue una tendencia homogénea en las subescalas. 


\section{Cuadro 2}

Media, desviación estándar, confiabilidad interna y matriz de intercorrelaciones

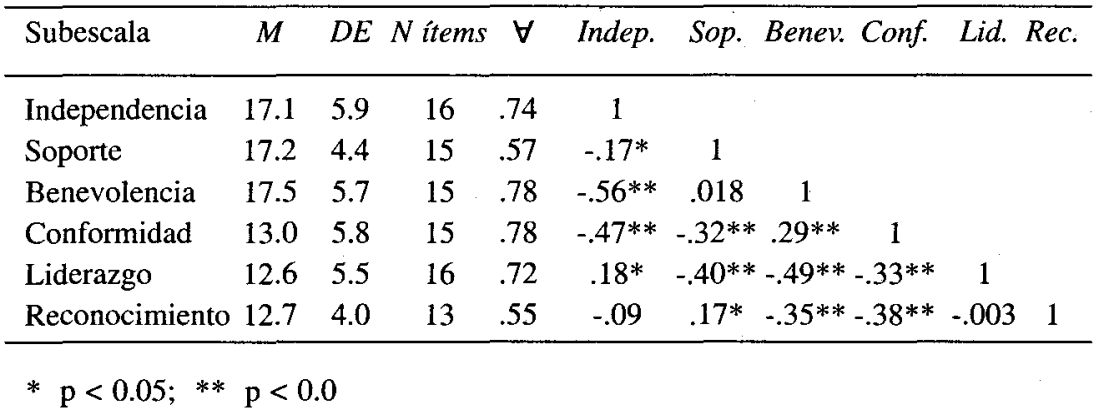

Ninguna de las intercorrelaciones en el Cuadro 2 son bastantes elevadas en su valor absoluto y más del $50 \%$ muestra signo negativo, lo cual es un fenómeno característico de medidas ipsativas (Loo, 1999). La correlación más fuerte es -.561, entre Independencia y Benevolencia. Esto sugiere una relación inversa, donde los puntajes de ambas tienden a moverse en direcciones opuestas. Hay otras correlaciones moderadamente fuertes, pero puede ser difícil, en este punto, interpretarlas de manera colectiva.

\section{Análisis de Componentes Principales}

En el Cuadro 3, vemos los primeros resultados del ACP. La magnitud de los eigenvalues va desde 2.273 hasta 0 . Las entradas bajo las columnas PCI, PCII,..., PCVI corresponden a los eigenvectores normalizados. El signo proporción (\%) se refiere a la proporción de la varianza explicada por los componentes individuales, y la última fila se refiere a la proporción acumulada de la varianza explicada. 
Evaluación de la estructura factorial del Cuestionario de Valores Interpersonales (SIV)

\section{Cuadro 3}

Análisis de componentes principales de las seis subescalas

\begin{tabular}{lcccccc}
\hline Subescala & $P C I$ & $P C I I$ & $P C I I I$ & $P C I V$ & $P C V$ & $P C V I$ \\
\hline Independencia & .47 & .16 & .60 & -.30 & -.24 & -.47 \\
Soporte & -.04 & -.69 & .27 & .17 & .55 & -.33 \\
Benevolencia & -.55 & .03 & .11 & .43 & -.54 & -.43 \\
Conformidad & -.47 & .35 & -.19 & -.50 & .37 & -.45 \\
Liderazgo & .42 & .38 & -.34 & .56 & .26 & -.41 \\
Reconocimiento & .25 & -.47 & -.62 & -.31 & .35 & -.31 \\
Eigenvalue & 2.27 & 1.55 & .95 & .69 & .52 & 0.0 \\
$\%$ & .37 & .25 & .15 & .11 & .08 & 0 \\
$\%$ acumulado & .379 & .637 & .79 & .912 & 1.0 & 1.0 \\
\hline
\end{tabular}

Los valores $Z$ (componentes) se asumen como no correlacionados $y$, en el Cuadro 3 , podemos ver que los primeros tres componentes principales explican una proporción acumulada de

$$
\frac{\operatorname{Var}\left(Z_{1}\right)+\operatorname{Var}\left(Z_{2}\right)+\operatorname{Var}\left(Z_{3}\right)}{6}=\frac{2.2733+1.5504+.9563}{6}=.797
$$

de la varianza total. Aquí, la varianza total está definida como la suma de los elementos diagonales de R (la matriz de correlaciones) $o$, de manera equivalente, la suma de las varianzas muestrales de los valores estandarizados en las subescalas. Aunque la tradicional regla de elegir los componentes con eigenvalue mayor o igual a 1.0 (Nunnally \& Bernstein, 1995) sugiere solo dos componentes, la relevancia del tercer componente es meritoria de análisis.

Considerando el método ipsativo de respuesta, es necesario interpretar el componente principal asociado con el eigenvalue más pequeño $(0)$. Un eigenvalue de 0 (varianza de la muestra $=0$ ) nos advierte que hay una dependencia lineal exacta de los datos. Es decir, una variable puede estar descrita como una combinación lineal de las restantes variables. Debido a que los coeficientes en cada una de las variables 
en el componente $Z_{6}$ son todos del mismo signo y, aproximadamente, del mismo tamaño, podemos concluir que la suma de Independencia, Soporte, Benevolencia, Conformidad, Liderazgo y Reconocimiento debe ser una constante. En efecto, el examen de nuestros datos muestra que la suma de todos los ítems de cualquiera de las escalas es siempre 90.

Una dependencia lineal exacta puede causar dificultades en los análisis subsecuentes. Por ejemplo, un cálculo que requiera la inversa de la matriz de correlación (o covarianza) es imposible, ya que tal inversa no puede ser calculada. Para quebrar tal dependencia lineal, decidimos remover la subescala Reconocimiento de los análisis posteriores, tal como también lo recomienda Loo (1999) al factorizar datos ipsativos. Entre Reconocimiento e Independencia, la primera se eligió al azar. Esto se determinó debido que ambas tenían las más altas cargas factoriales y de signo opuesto entre ellas, y, por lo tanto, tendían a contribuir fuertemente a la dependencia lineal.

Se efectuó nuevamente un ACP en la matriz de correlaciones R, considerando sólo las subescalas Independencia, Apoyo, Benevolencia, Conformidad y Liderazgo. Los primeros tres componentes principales con la solución rotada por el criterio varimax y sus varianzas se muestran a continuación en el Cuadro 4 . Se debe notar que el eigenvalue más pequeño en este componente es .0905 , así que aún hay una cercana dependencia lineal entre Independencia, Soporte, Benevolencia, Conformidad y Liderazgo. 
Evaluación de la estructura factorial del Cuestionario de Valores Interpersonales (SIV)

\section{Cuadro 4}

Análisis de componentes principales para $m=5$ y $m=3$, con rotación varimax

\begin{tabular}{|c|c|c|c|c|c|c|c|c|c|}
\hline \multirow[b]{2}{*}{ Variable } & \multicolumn{5}{|c|}{$m=5$} & \multicolumn{4}{|c|}{$m=3$ (con rotación várimax) } \\
\hline & $P C 1$ & $P C 2$ & $P C 3$ & $P C 4$ & $P C 5$ & $P C 1$ & $P C 2$ & $P C 3$ & $h^{2}$ \\
\hline Independencia & .52 & -.08 & .66 & .25 & .46 & -.97 & .01 & -.003 & .94 \\
\hline Soporte & -.12 & -.78 & -.18 & -.35 & .45 & .13 & -.31 & .89 & .90 \\
\hline Benevolencia & -.54 & .00 & -.11 & .73 & .38 & .70 & -.41 & -.08 & .67 \\
\hline Conformidad & -.43 & .49 & .29 & -.52 & .45 & .41 & -.37 & -.70 & .81 \\
\hline Liderazgo & .46 & .36 & -.64 & -.00 & .48 & -.15 & .97 & -.11 & .97 \\
\hline Eigenvalue & 2.196 & 1.36 & 0.75 & 0.58 & 0.09 & 1.65 & 1.35 & 1.31 & 4.32 \\
\hline$\%$ & 0.43 & 0.27 & 0.15 & 0.11 & 0.01 & .33 & .27 & .26 & .86 \\
\hline$\%$ acumulado & 0.43 & 0.71 & 0.86 & 0.98 & 1.0 & & & & \\
\hline
\end{tabular}

Estos componentes acumulan una proporción de $(2.1966+1.3682+.7559) / 5=.864$, o $86.4 \%$ de la varianza estandarizada total.

La magnitud y el signo de los coeficientes pueden ayudarnos a interpretar los componentes hallados. Por ejemplo, el componente $Z_{1}$ para la solución con $m=5$ tuvo coeficientes positivos relativamente grandes en Independencia y Liderazgo, y coeficientes negativos relativamente grandes en Benevolencia y Conformidad. El coeficiente de Soporte es pequeño y puede ser ignorado. Este componente representa un contraste entre las subescalas Independencia y Liderazgo, y por otro lado, Benevolencia y Conformidad.

Los tres componentes principales explican cerca del $86 \%$ de la varianza total de la muestra. Es decir, la variabilidad de la muestra es resumida bastante bien por los tres componentes, y la reducción de cinco variables (subescalas) a tres variables (componentes) reporta solo una pérdida de $14 \%$ de la varianza total; por lo tanto tal reducción es aceptable. 
Los resultados para Independencia, Soporte, Benevolencia, Conformidad y Liderazgo considerando $m=3$ factores comunes y la rotación varimax se muestran en el Cuadro 4.

Cuando se factorizan correlaciones, se considera que la carga es grande si su valor absoluto es igual o mayor a .4; las cargas menores a .4 se ignoran (se los toman como cero) en la interpretación de los factores comunes.

Con una solución como la encontrada, las correlaciones residuales parecen ser razonablemente pequeñas. Dados la matriz residual y el $86.4 \%$ de la varianza total que nuestro modelo explica (ver el último número en la fila \% Var en el Cuadro 2), podemos concluir que un modelo de $m=3$ factores es un buen ajuste a los datos.

Las cargas rotadas en el Cuadro 4 descubren una configuración bipolar de relaciones entre las subescalas. El primer factor parece representar un contraste entre Independencia y Benevolencia, de tal modo que los adolescentes con elevados puntajes en este factor tendrán altos puntajes en Independencia, mientras que los alumnos con elevados puntajes en Benevolencia tendrán puntajes en promedio bajos en el factor. Esto es un factor bipolar. El segundo factor es dominado por Liderazgo. El tercer factor representa un contraste entre Soporte y Conformidad. La interpretación de este factor bipolar es igual que el primer factor. Estas relaciones bipolares pueden representar un artificio creado por la ipsatividad del formato de respuesta del SIV.

\section{Análisis Factorial}

¿Los atributos medidos por Independencia, Soporte, Benevolencia, Conformidad y Liderazgo, son diferentes? Si es así, un análisis factorial confirmatorio con $m=p$, el número de variables igual al número 
de factores, debería mostrar que cada variable carga altamente sobre un único factor y tiene, a la vez, cargas insignificantes en los restantes factores. El Cuadro 5 contiene los resultados del análisis con $m=5$.

\section{Cuadro 5}

Análisis factorial para cinco subescalas, $m=5$, rotación varimax

\begin{tabular}{lcccccc}
\hline Subescala & $P C I$ & $P C I I$ & $P C I I I$ & $P C I V$ & $P C V$ & $h^{2}$ \\
\hline Independencia & .13 & -.29 & .90 & -.26 & -.01 & 1.0 \\
Soporte & -.94 & -.02 & -.11 & -.20 & .22 & 1.0 \\
Benevolencia & .30 & .91 & -.29 & .08 & .26 & 1.0 \\
Conformidad & .22 & .09 &.-.25 & .91 & .19 & 1.0 \\
Liderazgo & .25 & -.26 & .012 & -.20 & -.90 & 1.0 \\
Eigenvalue & 1.02 & 1.00 & .99 & .99 & .98 & 5 \\
$\%$ & .20 & .20 & .19 & .19 & .19 & 1 \\
\hline
\end{tabular}

En el Cuadro 5 se ve que las variables cargan por sobre .89. La comunalidad para cada variable es 1; es decir, la estructura del SIV se sustenta en un modelo ortogonal bien definido, demostrándose que cada variable es un factor. Este modelo reproduce exactamente la matriz de correlación $\mathrm{R}$ que en nuestra muestra se ha obtenido. Obsérvese también que los cambios en el monto de varianza explicada por cada factor realmente no difieren ni moderadamente.

\section{Conclusiones}

Nuestros resultados proporcionaron la evidencia sobre la ortogonalidad de las escalas del Cuestionario de Valores Interpersonales de Gordon (SIV) y de los efectos del método ipsativo de respuesta en las correlaciones. En base al análisis factorial, como se hipotetizó, cada variable observable (subescala) cargó altamente sobre un solo factor, mientras que mantuvo cargas verdaderamente insignificantes sobre las restantes. Tal como se sugiere para la construcción de los instrumentos de medición, la simplicidad factorial es una característi- 
ca deseable que tiene también efectos relevantes para la interpretación de los puntajes y su validez (Fleming, 1985). Con el análisis de componentes principales se reveló una estructura correlacional y con el análisis factorial especificando el número de factores se pudo comprobar el modelo bajo el cual se ha construido el SIV.

Estos resultados pueden dar un "alivio" sólo temporal a los usuarios del SIV, que lo han utilizado especialmente con adolescentes. Recalcamos que el presente estudio utilizó una muestra de adolescentes de niveles socioeconómicos diferenciados (medio y bajo) por el colegio y zona de residencia en que estudiaban. Pero dada la robustez del SIV, podría ser esperable la invarianza factorial en otras muestras que difieran en edad. Con las cargas factoriales bastante elevadas y comunalidades iguales a la unidad, se puede tener un amplio margen de certeza sobre la replicabilidad de resultados como los nuestros (Guadalogni \& Velicer, 1988) y, por lo tanto, se puede afirmar confiablemente un modelo de valores interpersonales claramente ortogonales. Pero de hecho, la generalización solo será asegurada con posteriores estudios psicométricos.

Las correlaciones entre los factores, ahora analizadas desde el análisis de componentes, han mostrado un patrón de correlaciones que, sin embargo, pueden provenir de una artificialidad que comúnmente ocurre en instrumentos ipsativos y cuyo signo característico es el surgimiento de factores bipolares y correlaciones negativas; esto converge con la literatura que ha abordado este problema (Cornwall \& Dunlap, 1994; Weiss, 1970, 1971).

Sin embargo, el patrón de relaciones bipolares no tiene que ser constante en otras muestras. Por ejemplo, en Bouvard et al. (1999) Soporte $(f=-.66)$, Independencia (-.52) y Conformidad (.60) cargaron sobre un componente, formando lo que parece ser el componente Conformismo. Adicionalmente, otro componente hallado estuvo construido por cargas negativas en Conformidad (-.44), Benevolencia 
(-.82), y cargas positivas en Reconocimiento (.74), Liderazgo (.47), que según Bouvard y colaboradores definen el componente llamado Altruismo. Cabe advertir que Bouvard et al. (1999) factorizaron las subescalas del SIV con otras medidas de variables interpersonales y en una muestra de adultos, y no relevaron en su interpretación el hecho de la ipsatividad del SIV.

La interpretación clínica y conceptual del SIV, tal como otras medidas ipsativas, tiene una importante distinción que ha sido suficientemente reconocida por los usuarios y revisores de pruebas. Esto es, que las interpretaciones que se extraen de los resultados muestran variabilidades intra-sujeto más que de tipo normativo o entre-sujetos (Gregory, 2000; Weiss, 1970). Recordemos que las pruebas referidas a normas permiten comparaciones normativas del desempeño del sujeto. Pero con medidas como el SIV, la magnitud de un puntaje (un valor interpersonal) es relativo a la magnitud del puntaje en otros valores interpersonales; esto proviene del método ipsativo para responder a un ítem de una subescala sobre otras. De aquí emerge un aspecto contradictorio que también ocurre en pruebas como el Cuestionario de Preferencias Personales de Edwards y el Inventario de Estilos de Aprendizaje de Kolb, que es la provisión de puntaje estandarizado y percentiles para una interpretación de naturaleza normativa o entresujetos. Esta metodología, de combinar un procedimiento de calificación ipsativo con una interpretación referidas a normas, es un aspecto de confusión debido a su incompatibilidad (Gregory, 2000).

Aún con estas características, el SIV sigue siendo utilizado y es frecuentemente ubicado como criterio en la exploración de atributos interpersonales (Bouvard et al., 1999; Durand, 1996). Aunque también posee elementos aceptables no compartidos con algunos cuestionarios de personalidad, como el no traslapamiento de ítems con las subescalas y la estructura relativamente ortogonal de sus factores subyacentes, la prueba puede mejorar si se incluye una "deipsativación", como separar las opciones convirtiéndolas en ítems independientes (Weiss, 1971; 
Marshall \& Merritt, 1986) o colocar estimaciones de confiabilidad en el vector diagonal de la matriz de correlaciones reducida (Weiss, 1971). Por otro lado, la solución de eliminar una subescala en el análisis no corrige las anormalidades surgidas, como es la alta proporción de intercorrelaciones; esto también ha sido confirmado por Loo (1999) y Cornwall y Dunlap (1994).

A la luz de los presentes resultados, y con las ventajas y desventajas de las medidas ipsativas, el SIV ha probado contener una estructura subyacente de factores que explican una parte de la configuración de los valores interpersonales en adolescentes. Un aspecto que nos alerta son las estimaciones marginalmente aceptables y bajas de la confiabilidad interna. La confiabilidad por medio del método de Cronbach (1951) ofrece el límite inferior de la confiabilidad interna (Muñiz, 1998), así que de otras estimaciones alternativas se pueden obtener valores más benevolentes. Se ha reportado previamente que incluso las estimaciones de confiabilidad son afectadas por la ipsatividad de una medida (Loo, 1999). Sin embargo, un análisis sobre los componentes de variabilidad en el SIV, por ejemplo desde la teoría de la generalizabilidad (Brennan, 1983), puede ofrecer un análisis formal y elegante de estos niveles bajos de confiabilidad en muestras de adolescentes.

\section{Referencias}

Beck, A. T., Rush, A. J., Shaw B. F. \& Emery, G. (1979). Cognitive therapy of depression: A treatment manual. Nueva York: Guilford Press.

Bouvard, M., Arrindel, W. A., Guérin, J., Bourchard, C., Rion, A.,

Ducotter, E., Séchaud, M., Mollard, E., Grillet, P., Dang, C. \& Cottraux, J. (1999). Psychometric appraisal of the Scale for Interpersonal Behavior (SIB) in France. Behaviour Research and Therapy, 37, 741-762. 
Evaluación de la estructura factorial del Cuestionario de Valores Interpersonales (SIV)

Brennan, R. L. (1983). Elements of Generalizability Theory. Iowa: American College Testing.

Castillo, B. (1977). Estudio y tipificación del Cuestionario de Valores Interpersonales de L. V. Gordon en estudiantes de Cajamarca, Huancayo y Cusco. Tesis de bachiller no publicada, Universidad Nacional Mayor de San Marcos, Lima.

Cornwall, J. M. \& Dunlap, W. P. (1994). On the questionable soundness of factoring ipsative data: A response to Saville and Willson. Journal of Occupational and Organizational Psychology, 67, 89-100.

Crocé, R. (1985). Grado de dogmatismo y valores interpersonales en un grupo de estudiantes universitarios pertenecientes a diferente nivel socioeconómico y sexo. Tesis de licenciatura no publicada, Universidad San Martín de Porres, Lima.

Cronbach, L. J. (1951). Coefficient alpha and the internal structure of tests. Psychometrika, 16(3), 297-334.

Cronbach, L. J. \& Meehl, P. E. (1955). Construct validity in psychological tests. Psychological Bulletin, 52, 281-302.

Cudeck, R. (2000). Exploratory factor analysis. En H. E. A. Tinsley \& S. D. Brown (Eds.), Handbook of multivariate statistics and mathematical modeling (pp. 265-296). Nueva York: Academic Press.

Deluty, R. H. (1979). Children's Action Tendency Scale: A self-report measure of aggressiveness, assertiveness, and submissiveness in children. Journal of Consulting and Clinical Psychology, 47(6), 1061-1071.

Durand, A. (1996). Ambiente familiar y valores en estudiantes del 1er. al 5to. de secundaria. Tesis de licenciatura no publicada, Universidad Nacional Federico Villareal, Lima.

Fabrigar, L. R., MacCallum, R. C., Wegener, D. T. \& Strahan, E. J. (1999). Evaluating the use of exploratory factor analysis in psychological research. Psychological Methods, 4(3), 279-299.

Fleming, J. S. (1985). An index of fit for factor scales. Educational and Psychological Measurement, 45, 725-728. 
Gall, M. D., Borg, W. R. \& Gall, J. P. (1996). Educational research: An introduction (6a. ed.). White Plains, NY: Longman Publishers.

Gordon, L. V. (1993). Survey of interpersonal values, SRA, Examiners manual (3a. ed.). Londres: MacMillan.

Gregory, R. J. (2000). Psychological testing: History, principles, and applications (3a. ed.). Boston: Allyn \& Bacon.

Guadalogni, E. \& Velicer, W. (1988). Relation of sample size to the stability of component patterns. Psychological Bulletin, 103(2), 265-275.

Guilford, J. P. (1952). When not to factor analyze. Psychological Bulletin, 49, 26-37.

Heise, D. R. \& Bohrnstedt, G. W. (1970). Validity, invalidity, and reliability. En E. E. Borgata \& G. W. Bohrnstedt (Eds.), Sociological methodology (pp. 104-129). San Francisco: Jossey-Bass.

Helms, J. E. (1983). A practitioner's guide to the Edwards Personal Preference Schedule. Springfield, IL: Charles C. Thomas.

Hutcheson, G. \& Sofroniou, N. (1999). The multivariate social scientist. Londres: Sage.

Johnson, R. A. \& Wichern, D. W. (2002). Applied multivariate statistical analysis (5a. ed.). Upper Saddle River, NJ: Prentice Hall.

Kincannon, J. C. (1968). Prediction of the standard MMPI scale scores from 71 items: The Mini-Mult. Journal of Consulting and Clinical Psychology, 32, 319-325.

Kolb, D. A. (1976). Learning Style Inventory: Technical manual. Boston, MA: McBer \& Company.

Loo, R. (1999). Issues in factor-analyzing ipsative measures: The Learning Style Inventory (LSI-1985) example. Journal of Business and Psychology, 14(1), 149-154.

Marks, S. I. \& Mathews, M. (1979). Brief standard self-rating for phobic patients. Behavior Research and Therapy, 17, 263-267.

Marshall, J. C. \& Merritt, S. L. (1986). The Learning Styles Questionnaire: A validity study. Educational and Psychological Measurement, 46, 257-262. 
Evaluación de la estructura factorial del Cuestionario de Valores Interpersonales (SIV)

May, R. B., Masson, M. J. \& Hunter, M. A. (1990). Application of statistics in behavioral research. Nueva York: Harper \& Row.

Muñiz, J. (1998). Teoría clásica de los test. Madrid: Pirámide.

Nunnally, J. C. \& Bernstein, I. J. (1995). Teoría psicométrica (3a. ed.). México, DF: McGraw Hill.

Nuñez, R. (1973). Valores en las relaciones interpersonales de estudiantes de diferente nivel socioeconómico. Tesis de bachiller no publicada, Universidad Nacional Mayor de San Marcos, Lima.

Rivas, K., Wichern, D. \& Merino, C. (2002). Una mirada multivariada a los valores interpersonales. Manuscrito en preparación.

Saville, P. \& Willson, E. (1991). The reliability and validity of normative and ipsative approaches in the measurement of personality. Journal of Occupational and Organizational Psychology, 64, 219-238.

Silva, M. (1982). Estudio comparativo del grado de dogmatismo y valores interpersonales de los comuneros residentes del Valle del Mantaro (Sicaya y San Jerónimo) en relación a los migrantes. Tesis de Licenciatura no publicada, Universidad San Martín de Porres, Lima.

Van Gelderen, M. \& Frese, M. (1998). Strategy process as a characteristic of small scale business owners: Relationships with success in a longitudinal study. En P. D. Reynolds, W. D. Bygrave, N. M. Carter, S. Manigart, C. M. Mason, G. D. Meyer et al. (Eds.), Frontiers of entrepreneurship research (pp. 234248). Babson Park, MS: Babson College.

Vela, B. (1989). Cohesión y adaptabilidad familiar en el consumidor de pasta básica de cocaína. Tesis de bachiller no publicada, Universidad Peruana Ricardo Palma, Lima.

Velicer, W. F. \& Jackson, D. N. (1990). Component analysis versus common factor analysis: Some issues in selecting an appropriate procedure. Multivariate Behavioral Research, 25(1), 1-28.

Wegener, D. T. \& Fabrigar, L. R. (2000). Analysis and design for non experimental data: Addressing causal and non causal hypotheses. En H. T. Reiss \& C. M. Judd (Eds.), Handbook of research 
methods in social and personality psychology (pp. 412-450). Nueva York: Cambridge University Press.

Weiss, D. J. (1970). Factor analysis and counseling research. Journal of Counseling Psychology, 17(5), 477-485.

Weiss, D. J. (1971). Further considerations in applications of factor analysis. Journal of Counseling Psychology, 4, 85-92.

Worrell, F. C., Vandiver, B. J. \& Watkins, M. W. (2001). Construct validity of the Learning Behavior Scale with an independent sample of students. Psychology in Schools, 38(3), 207-215. 